\title{
Arranque de un motor de inducción usando control difuso
}

\section{Starting an induction motor with a fuzzy controller}

\section{Camilo Barriga Turriago}

Ingeniero eléctrico, ingeniero de I.M.MAN.COL. Bogotá, Colombia.kamilobt@, gmail.com

\section{JUAN ZAMBRANO}

Licenciado en Matemáticas y Física, especialista en Educación Matemática. Docente de la Universidad Distrital Francisco José de Caldas. Bogotá, Colombia. juanchoza@gmail.com

Fecha de recepción: 28 de mayo de 2011

Clasificación del artículo: Investigación (Conciencias)

Fecha de aceptación: 29 de agosto de 2011

Palabras clave: Arranque suave, control directo de par, inversor trifásico, lógica difusa, motor de inducción, tiristores.

Key words: Soft start, direct torque control, three-phase inverter, fuzzy logic, induction motor, thyristor.

\section{RESUMEN}

Este artículo presenta el aprovechamiento de la lógica difusa como parte de la inteligencia artificial en el área de electrónica de potencia y drivers de motores, para mejorar el desempeño durante el arranque de un motor de inducción. Para alimentar el motor de inducción, dos configuraciones de circuitos se han escogido: series de tiristores conectados en anti-paralelo y un inversor trifásico. Estrategias de control como arranque suave y Control Directo del Par incorporando control difuso han sido propuestas para reducir la corriente y mejorar el torque. Los resultados obtenidos de la simulación de un motor de inducción jaula de ardilla de $1.1 \mathrm{KW}$ con estos métodos electrónicos, muestran un mejoramiento en el desempeño en el periodo de arranque, reduciendo la corriente e incrementando el torque.

\section{ABSTRACT}

This paper presents the use of fuzzy logic as part of artificial intelligence in the area of power electronics and motor drivers to improve performance during the startup of an induction motor. To feed the induction motor, two circuit configurations have been chosen to use: a series of 
thyristors connected in ant parallel and a threephase inverter. Control strategies such as soft start and Direct Torque Control incorporating fuzzy control have been current proposals to reduce and improve torque. The results of a simulated induction motor squirrel cage of $1.1 \mathrm{KW}$ to these electronic methods show an improvement in performance at boot time, reducing power and increasing the torque.

\section{INTRODUCCIÓN}

Los motores de inducción son de gran importancia y de extendido uso en la industria por sus múltiples ventajas: su fiabilidad, bajo costo, una estructura simple, un mantenimiento práctico y su larga vida. Aunque estos motores tienen muchas características favorables, también poseen no linealidad e interacciones dinámicas que varían en el tiempo [1]. Además los motores de inducción durante el proceso de arranque, la corriente instantánea es tan grande que usualmente alcanza cuatro o siete veces la corriente nominal, experimentando con esto una gran sacudida sobre su torque inicial; esto tiene un impacto sobre la vida del motor, deteriorándolo y originando perturbaciones en la red eléctrica. Estas perturbaciones pueden llegar a ocasionar fallas en otras cargas de la misma red interna, afectando los procesos de producción de las industrias. Teniendo en cuenta estos fenómenos que se presentan en el arranque de los motores de inducción se debe limitar la corriente de arranque [2]. En la actualidad, el control difuso ha tenido un amplio uso en muchos campos; principalmente el control difuso imita el control que se logra por la experiencia, este puede aproximarse al control humano sin necesidad de construir el modelo matemático exacto del sistema, haciéndolo apropiado para sistemas no lineales, como motores de inducción. La mayor contribución de este artículo está en el aprovechamiento de la lógica difusa para mejorar el desempeño durante el arranque de un motor de inducción jaula de ardilla, usando arranques electrónicos tales como el arranque por medio de tiristores y un inversor trifásico.

\section{LÓGICA DIFUSA}

La lógica difusa o teoría de conjuntos difusos ha emergido como una herramienta provechosa para el control de procesos industriales complejos, sistemas de diagnóstico y otros sistemas expertos. Fue introducida por el Dr. Lofti Zadeh de la universidad de Berkeley en 1965 como una herramienta matemática para tratar con incertidumbres.

La teoría difusa provee un mecanismo para representar conceptos lingüísticos tales como "mucho", "poco", "mediano", "bastante". En general, la lógica difusa suministra una estructura de inferencia que permite apropiar las capacidades del razonamiento humano [3].

Para establecer un control difuso, es necesario interpretar reglas que son basadas en la experiencia, como la símil causa y efecto en una acción. Estas reglas dan valores de entrada y salida correspondientes a situaciones de interés, mediante sentencias "SI... ENTONCES...", éstas son incorporadas.

Cuando se habla de Protocolo Lingüístico en un control difuso se habla de las sentencias o reglas que nos dan una descripción cualitativa del proceso, usando variables lingüísticas en vez de variables numéricas. El proceso de convertir una variable numérica a una variable lingüística (número difuso) se denomina fuzzificación. 


\section{con-ciencias}

La forma de obtener estas reglas depende del proceso y el conocimiento que se tenga acerca de este. Por ejemplo, una regla de control podría ser: si la salida es más baja que el requerimiento y está cayendo moderadamente, entonces, la entrada al sistema debe incrementarse gradualmente.

El proceso inverso de fuzzificación se denomina defuzzificación. El uso de controladores difusos proporciona una salida mediante una variable lingüística.

Acorde con el requerimiento del mundo real, las variables lingüísticas tienen que ser transformadas a salidas numéricas, uno de los métodos de defuzzificación más conocidos es el "centro de gravedad".

\section{METODOLOGÍA}

A continuación se describen el procedimiento realizado para el arranque suave difuso y el control directo de par difuso.

\subsection{Arranque suave difuso}

El uso de arranques suaves se mantiene como una solución tradicional en el arranque de motores de inducción para reducir la corriente. El principio de un arranque suave es fundamentalmente la variación del voltaje del motor hasta el valor nominal de operación, mediante el ajuste del ángulo de disparo de tiristores a frecuencia constante [4].

El ángulo $\alpha$ de disparo de los tiristores debe ser calculado como una función del tiempo [5]. En la Fig. 1 se muestra el circuito convencional del arranque suave, cada fase tiene dos tiristores que están conectados en anti paralelo. En esta configuración los tiristores son disparados de acuerdo con la secuencia de los pulsos de disparo como muestra la Fig. 2. En esta figura se puede observar que al menos dos tiristores deben conducir

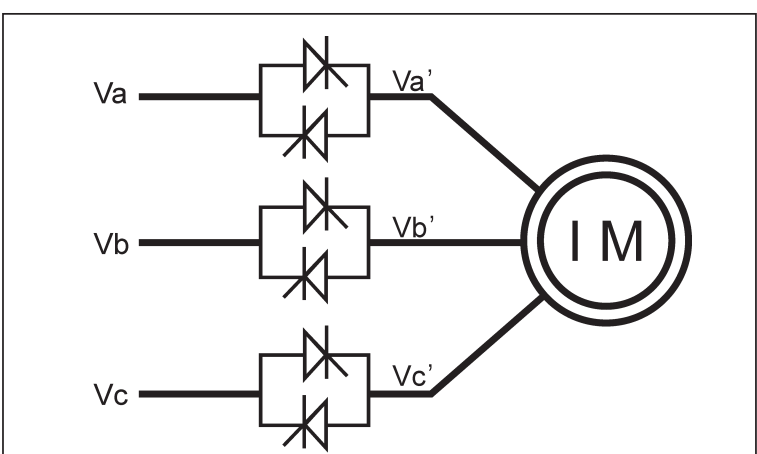

Fig. 1. Arranque por medio de tiristores.

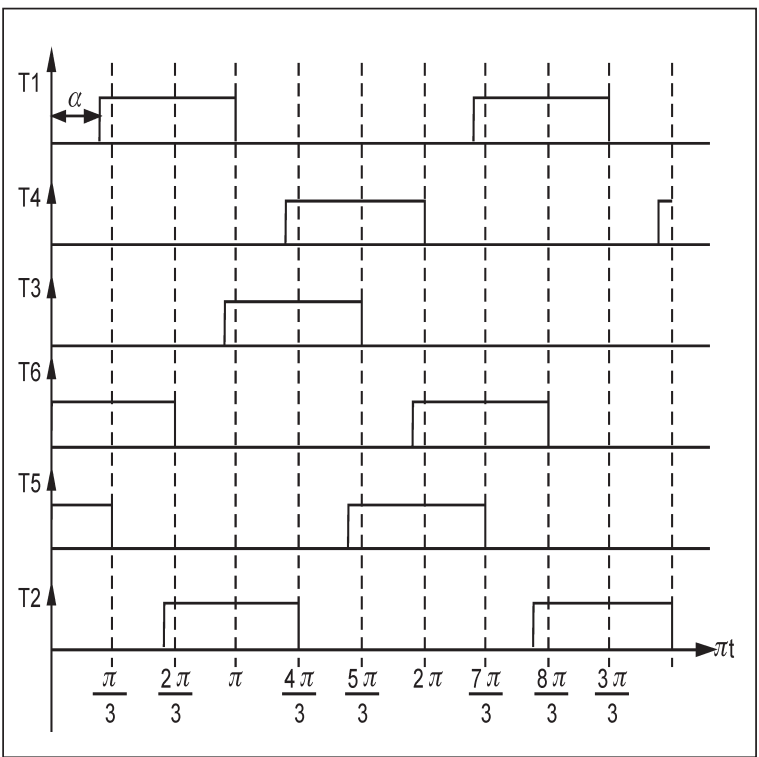

Fig. 2. Secuencia de disparo de tiristores.

simultáneamente para permitir el flujo de corriente hacia la carga [6].

Sin embargo, uno de los deméritos de un arranque suave es que dado a la relación cuadrática entre el par electromagnético y el voltaje, al reducir el voltaje se produce una considerable caída en el par electromagnético, el cual puede causar posibles fallas al motor con carga en el arranque [7]. Esto implica que el tradicional arranque suave es apropiado para aplicaciones de baja carga, a bajas velocidades, tales como ventiladores y bombas. 


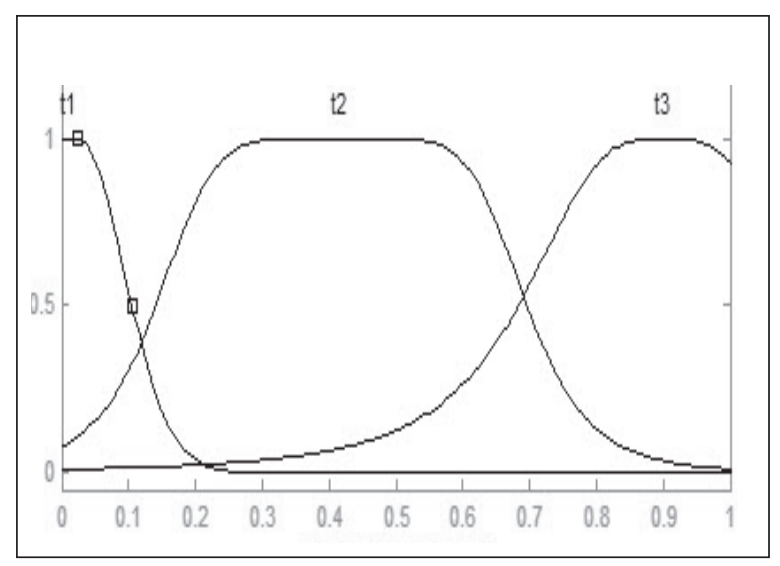

Fig. 3. Funciones de pertenencia propuestas para el tiempo de simulación.

\subsubsection{Control difuso}

El controlador difuso empleado es de tipo Mamdani para simular el algoritmo de arranque suave. Para el tiempo de simulación como señal de entrada del controlador, se fijaron tres funciones de pertenencia t1 (tiempo inicial), t2 (tiempo medio) y t3 (tiempo final). El ángulo $\alpha$ de disparo de los tiristores como la señal de salida del controlador se fijaron también tres funciones de pertenencia a1 (ángulo pequeño), a2 (ángulo medio) y a3 (ángulo grande).

Las Fig. 3 y 4 presentan las funciones de pertenencia propuestas de forma gaussiana para la

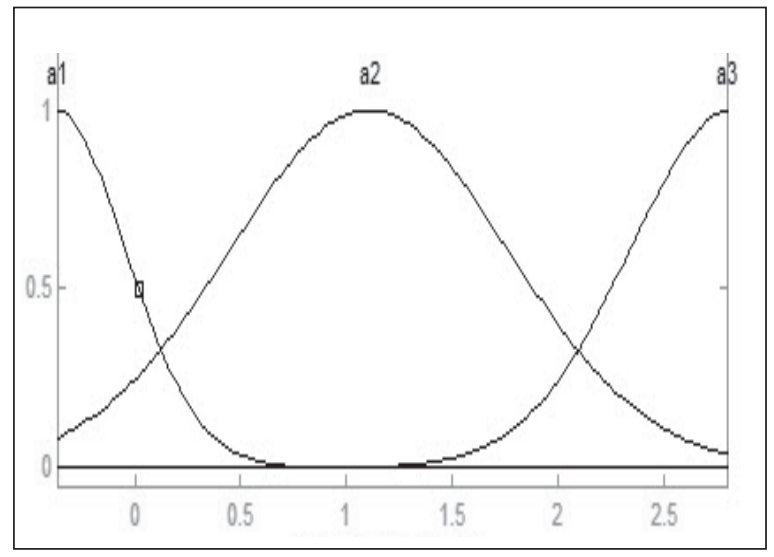

Fig. 4. Funciones de pertenencia propuestas para el ángulo de disparo.

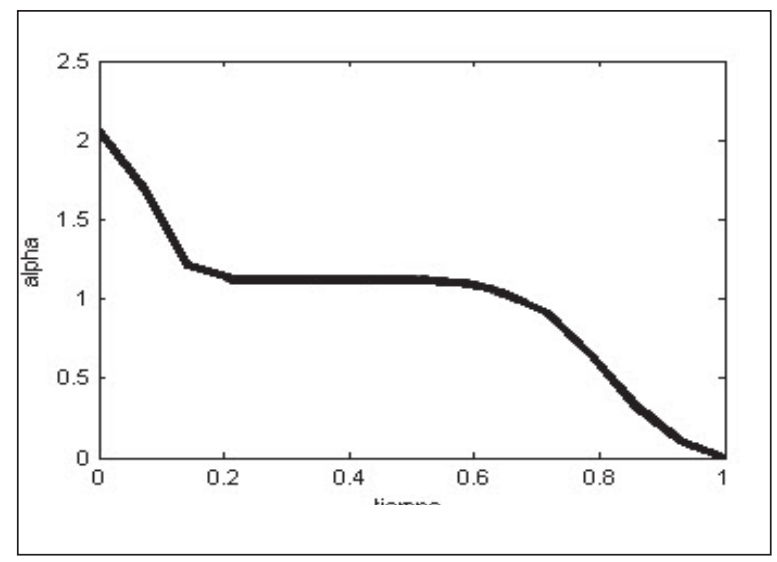

Fig. 5. Relación entre la entrada y la salida en el controlador difuso del arranque suave.

entrada y la salida del controlador respectivamente.

El controlador difuso es diseñado intuitivamente, los tiristores son inicialmente ( $\mathrm{t} 1$ ) disparados con un ángulo $\alpha$ grande (a3) de este modo el voltaje aplicado es pequeño. Secuencialmente el ángulo $\alpha$ va variando de $118^{\circ}(2.06 \mathrm{rad})$ a $0^{\circ}$ durante el arranque del motor de inducción, esta relación entre la entrada y la salida en el controlador difuso del arranque suave se muestra en la Fig. 5.

\subsection{Control directo de par (DTC) difuso}

Un control avanzado de máquinas eléctricas requiere un control independiente del flujo magnético y del par. El control directo de par se caracteriza por su fácil implementación y una respuesta dinámica rápida. Además, el inversor es directamente controlado por el algoritmo mismo sin necesidad de emplear una técnica de modulación. El diagrama de bloques del control propuesto se muestra en la Fig. 6. Este incorpora un controlador difuso para controlar la velocidad en el esquema básico del control directo del par (DTC).

La idea básica del DTC es calcular los valores instantáneos del par y del flujo, a partir de las variables del estator de la máquina. Estas variables 


\section{con-ciencias}

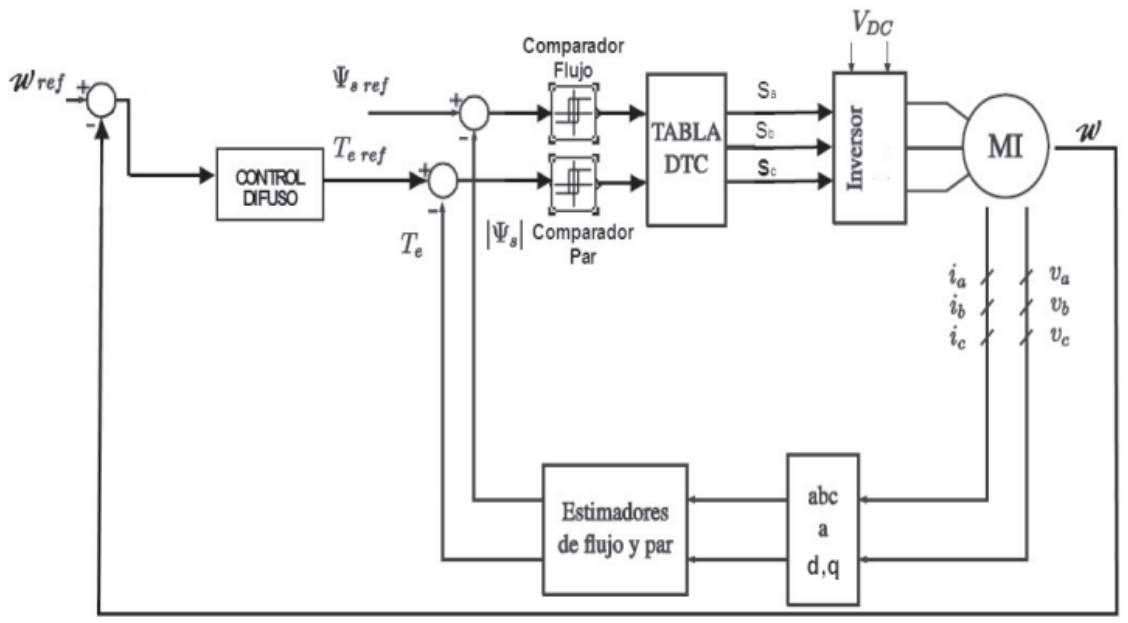

Fig. 6. Sistema de control propuesto DTC difuso.

de voltaje y corriente del estator son transformadas de coordenadas $a-b-c$ a un modelo referido a dos ejes ortogonales $q-d$ mediante,

El par y el flujo se controlan directamente y de forma independiente, mediante la selección óptima de los estados de conmutación del inversor y limitando los errores del flujo y del par, mediante controladores histéresis del flujo y del par [8].

$$
\left[\begin{array}{l}
V_{s d} \\
V_{s q}
\end{array}\right]=\left[\begin{array}{ccc}
1 & 1 / 2 & 1 / 2 \\
0 & \sqrt{3} / 2 & \sqrt{3} / 2
\end{array}\right]\left[\begin{array}{l}
V_{a} \\
V_{b} \\
V_{c}
\end{array}\right]
$$

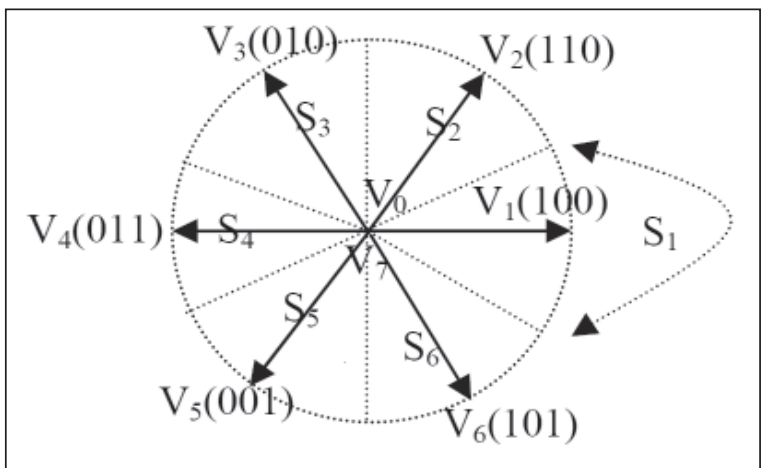

Fig. 7. Definición de los vectores espaciales de voltaje.
La estrategia del DTC es clara. El par del motor se controla efectivamente con la rotación del vector del flujo del estator utilizando estados de conmutación adecuados. En el mismo tiempo se controla la magnitud del vector del flujo del estator de la misma manera, es decir, con la utilización de estado de conmutación del inversor. Este valor se puede cambiar según los requisitos de la consigna del flujo. Los detalles del funcionamiento se pueden obtener con la ayuda de las Fig. 6 y 7.

Según la Fig. 6, los valores calculados del par y del flujo se comparan con sus consignas. Los errores entran en los controladores de histéresis. Sus salidas son señales lógicas de valores discre-

Tabla 1. Tabla de conmutación del inversor.

\begin{tabular}{|c|c|c|c|c|c|c|c|}
\hline \multirow{2}{*}{$\Psi$} & \multirow{2}{*}{$\mathrm{T}$} & \multicolumn{6}{|c|}{ Tabla de Conmutación } \\
\cline { 3 - 8 } & & S1 & S2 & S3 & S4 & S5 & S6 \\
\hline \multirow{2}{*}{1} & 1 & V5 & V6 & V1 & V2 & V3 & V4 \\
\cline { 2 - 8 } & 0 & V3 & V4 & V5 & V6 & V1 & V2 \\
\hline \multirow{2}{*}{0} & 1 & V6 & V1 & V2 & V3 & V4 & V5 \\
\cline { 2 - 8 } & 0 & V2 & V3 & V4 & V5 & V6 & V1 \\
\hline
\end{tabular}


tos 1,0 que se aplican a la tabla de conmutación que elige uno de los ocho estados posibles del vector espacial de la tensión del inversor [8].

\subsubsection{Estimadores de par y flujo}

El flujo del estator $\left(\bar{\psi}_{s}\right)$ desarrollado por el motor de inducción se estima mediante las ecuaciones (2), (3) y el par electromagnético $\left(R_{s} T_{\theta m}\right)$ se estima mediante la ecuación (4) [9].

$$
\begin{aligned}
& \bar{\psi}_{s d}=\int_{0}^{t}\left(\bar{V}_{s d}-R_{s} \bar{I}_{s d}\right) d t \\
& \bar{\psi}_{s q}=\int_{0}^{t}\left(\bar{V}_{s q}-R_{s} \bar{I}_{s q}\right) d t \\
& T_{e m}=p\left(\psi_{s d} I_{s d}-\psi_{s q} I_{s q}\right)
\end{aligned}
$$

Donde, $R_{s}$ es la resistencia del estator y $p$ es el número de pares de polos del motor.

\subsubsection{Control difuso}

El interés de incorporar un controlador difuso en el esquema de control directo de par, principalmente está en la facilidad de su implementación, que brinda un mejor desempeño que un control convencional PID [10].

Para la señal de entrada del controlador se tomó el error entre la referencia de velocidad y la velocidad del motor, fijándose para ésta tres funciones de pertenencia triangulares ZE (error cero), NS (error negativo pequeño), PS (error positivo pequeño) y dos funciones de pertenencia trapezoidales NL (error negativo grande), PL (error positivo grande).

Como salida del controlador difuso se tiene el par de referencia para el algoritmo de control directo del torque.

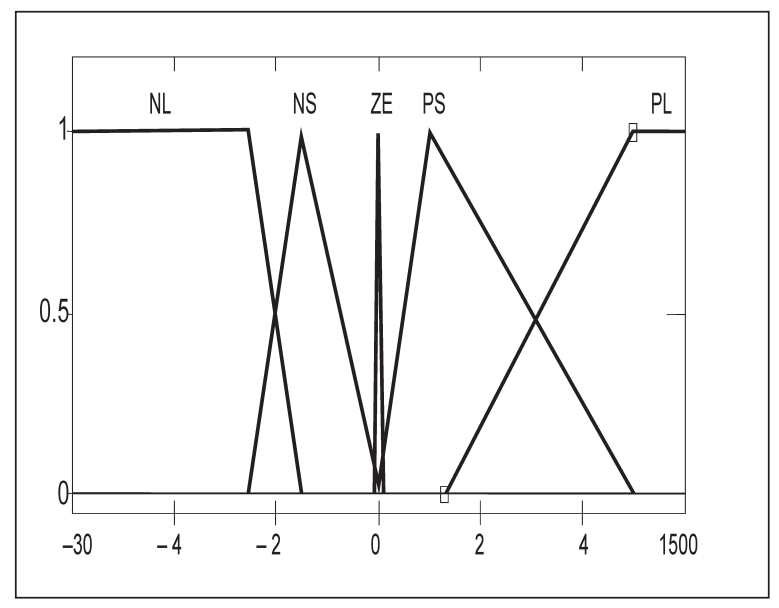

Fig. 8. Funciones de pertenencia propuestas para el Error.

Para esta salida se fijaron cuatro funciones de pertenencia triangulares TC (Par cero), TNP (Par negativo pequeño), TPP (Par positivo pequeño), TNG (Par negativo grande) y una función trapezoidal TPG (Par positivo grande), las Fig. 8 y 9 presentan las funciones de pertenencia para la entrada y salida del controlador respectivamente.

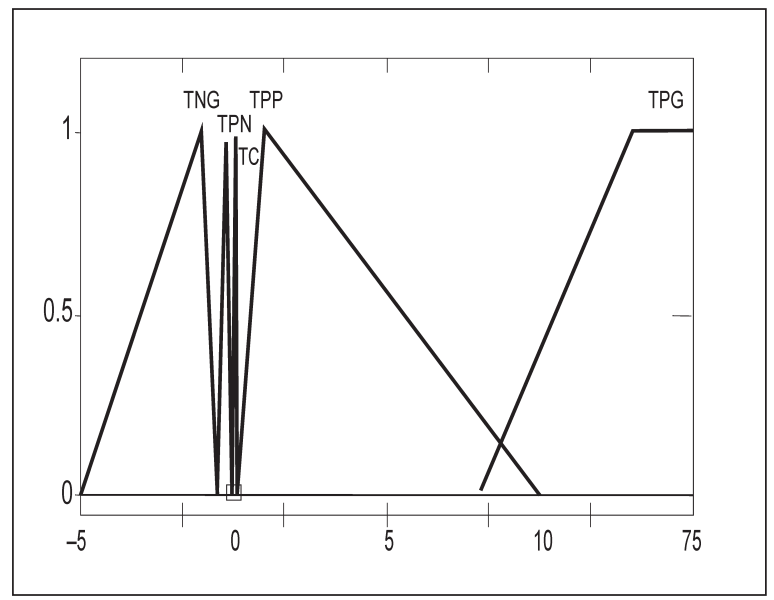

Fig. 9. Funciones de pertenencia propuestas para el Error.

El controlador difuso es diseñado con base en la observación de los resultados de la simulación, la forma y la posición de cada función de pertenencia se ajustaron mediante ensayo error. Las reglas del control difuso empleadas son como sigue, 


\section{con-ciencias}

1. Si error es NL entonces Par de referencia es TNG.

2. Si error es NS entonces Par de referencia es TNP.

3. Si error es ZE entonces Par de referencia es TC.

4. Si error es PS entonces Par de referencia es TPP.

5. Si error es PL entonces Par de referencia es TPG.

La inferencia difusa para evaluar estas reglas es del tipo Mamdani y centro de gravedad como método para la fuzzificación.

La relación entre la entrada y la salida en el controlador difuso para la regulación del par se presenta en la Fig. 10.

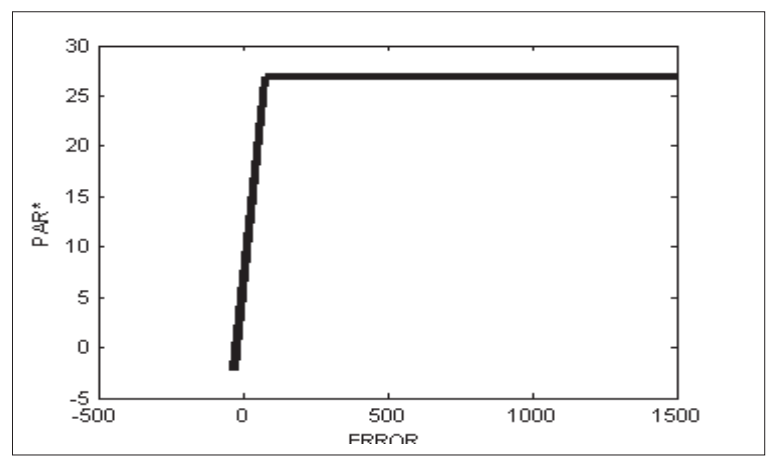

Fig. 10. Relación entre la entrada y salida en el controlador difuso.

\section{RESULTADOS}

A continuación se presentan los resultados de la simulación que fue llevada a cabo usando Matlab/ Simulink donde se muestran las variaciones de la corriente de arranque, velocidad y torque RMS durante el período de arranque, primero para un arranque directo, seguido por el arranque con tiristores usando la técnica arranque suave convencional, luego el arranque suave difuso y finalmente el arranque con el inversor trifásico y la técnica
Tabla 2. Parámetros del modelo eléctrico del motor de inducción.

\begin{tabular}{|l|c|}
\hline Parámetro & Valor $(\Omega)$ \\
\hline$R_{\underline{s}}$ (Resistencia del estator) & 3,5744555 \\
\hline$R_{r}$ (Resistencia del rotor) & 11,1375109 \\
\hline$X_{s}$ (Reactancia del estator) & 7,35384485 \\
\hline$X_{r}$ (Reactancia del rotor) & 7,35384485 \\
\hline$X_{m}$ (Inductancia mutua) & 237,837585 \\
\hline
\end{tabular}

Tabla 3. Características nominales del motor de inducción.

\begin{tabular}{|l|c|c|}
\hline Potencia nominal & kW & $\mathbf{1 , 1}$ \\
\hline Velocidad nominal & $\mathrm{r} / \mathrm{min}$ & 3350 \\
\hline Voltaje nominal d/y & {$[\mathrm{V}]$} & $220 / 380$ \\
\hline Frecuencia nominal & {$[\mathrm{Hz}]$} & 60 \\
\hline Corriente nominal d/y & {$[\mathrm{A}]$} & $4,7 / 2,7$ \\
\hline
\end{tabular}

control directo del par difuso. Los parámetros del motor de inducción empleados en las simulaciones se presentan en la tabla 2 y los datos de placa se presentan en la tabla 3 .

\subsection{Arranque directo}

Los resultados correspondientes de la simulación del arranque directo son ilustrados en la Fig. 9, ésta muestra que el valor de la corriente se eleva más de diez veces al valor de la corriente de estado estacionario. El rotor alcanza su velocidad síncrona en 0.6 segundos.

\subsection{Arranque Suave}

La Fig. 12 muestra cómo la corriente ha disminuido aproximadamente 6 veces el valor de la corriente de estado estacionario. También se puede observar que hay una disminución en el torque con gran impacto sobre la velocidad cuando el motor arranca con carga Fig. 13 (b), aquí se evi- 


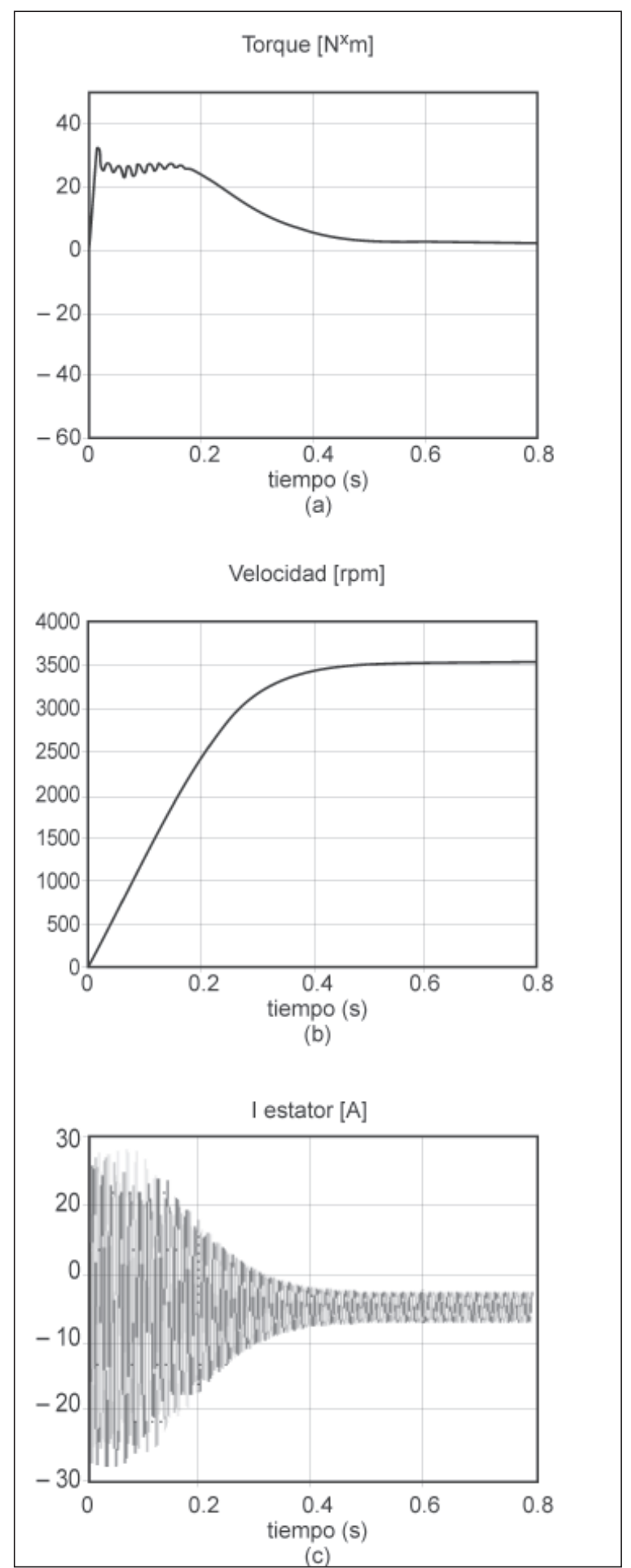

Fig. 11. Arranque directo en vacío. (a) Par, (b) Velocidad, (c) corriente.

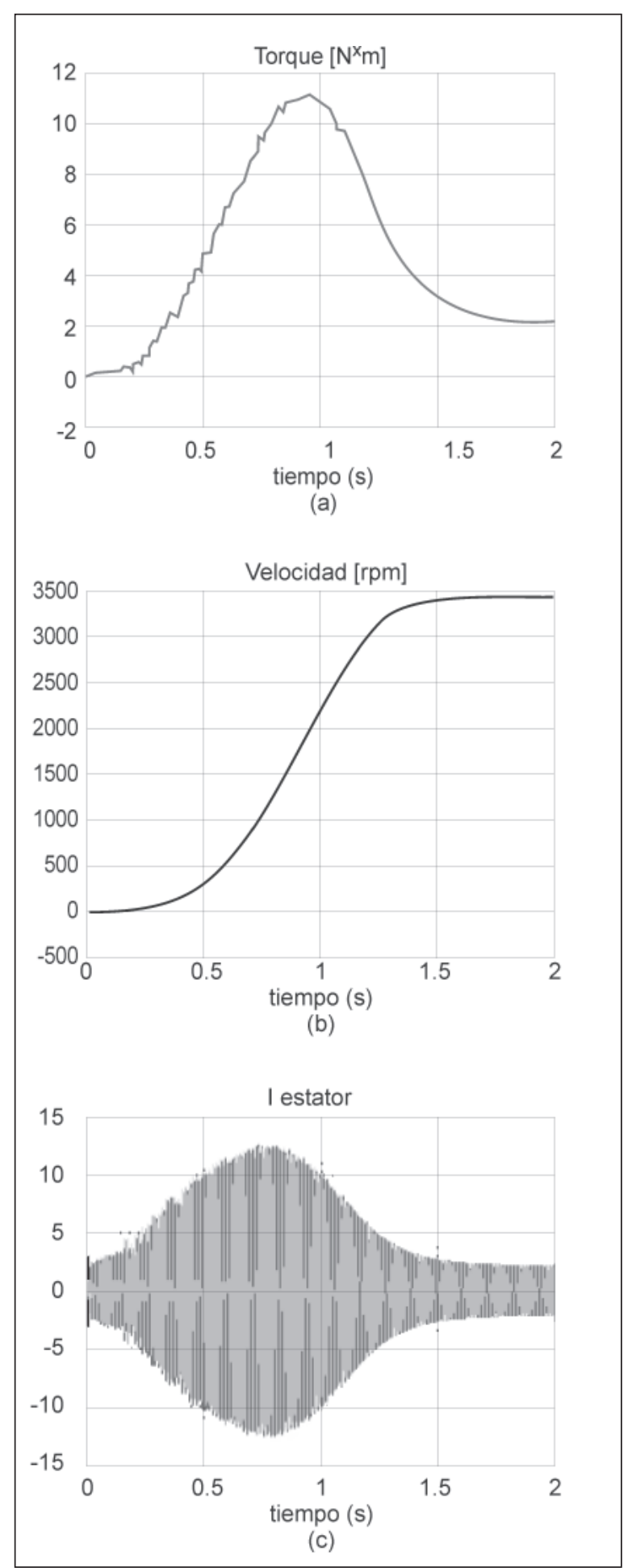

Fig. 12. Arranque suave en vacío (a) Par, (b) Velocidad, (c) corriente. 


\section{con-ciencias}

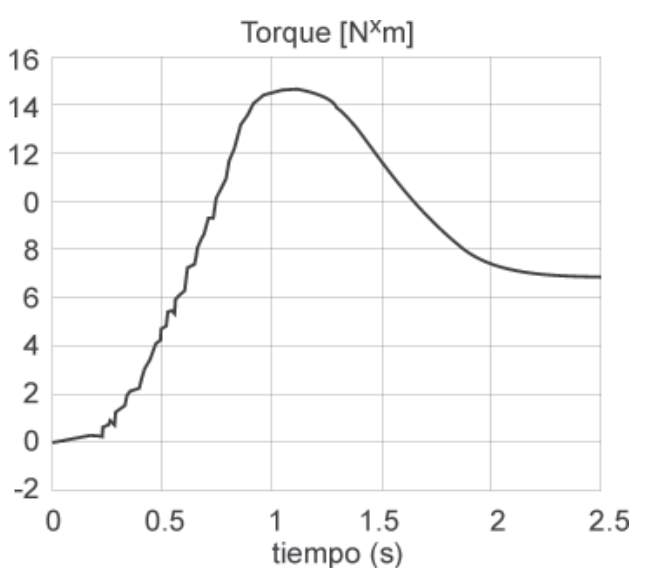

(d)

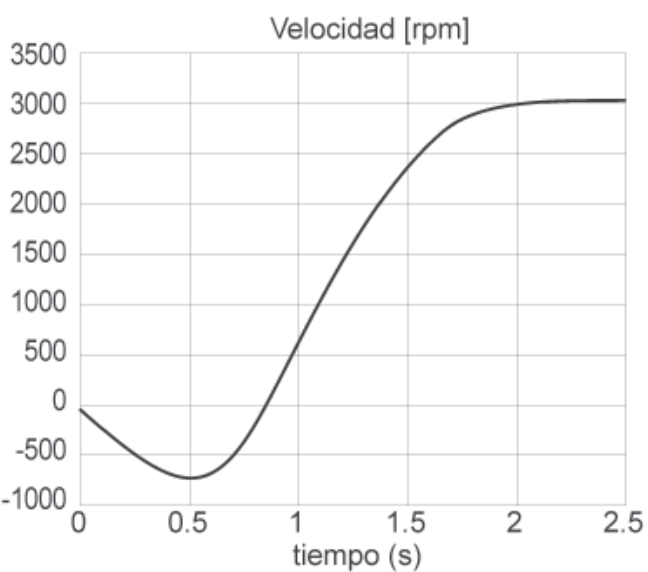

(e)

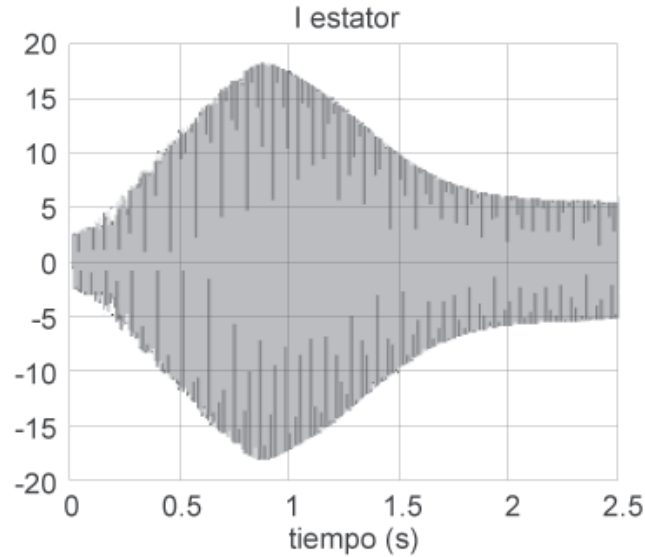

(f)

Fig. 13. Arranque suave en vacío con carga $5 \mathrm{~N} \mathrm{~m} \mathrm{(a)}$ Par, (b) Velocidad, (c) corriente. dencia que cerca de un segundo le toma al motor poder arrancar.

\subsection{Arranque suave difuso}

Aunque la corriente es un poco más elevada (aproximadamente 7 veces el valor de la corriente de estado estacionario) que el arranque suave convencional, la Fig. 14 muestra cómo el control difuso ha mejorado el par, incrementándolo y permitiéndole arrancar al motor con carga en un menor tiempo, sin un gran impacto sobre la velocidad como el que se evidenció en el arranque suave convencional.

El aumento en la corriente del arranque suave difuso queda compensado con el de mejoramiento que se logra en el par con relación al arranque suave convencional, al tener un mejor par y en un menor tiempo logra un arranque más rápido.

\subsection{Arranque con el inversor trifásico y DTC difuso}

La simulación con el inversor trifásico fue realizada intencionalmente para permitir la comparación con la técnica arranque suave convencional y el arranque suave difuso. La Fig. 15 muestra que en el arranque del motor de inducción hay una importante reducción en la corriente de arranque, con un par elevado y con menos oscilaciones.

El arranque con el inversor trifásico y DTC difuso le permite arrancar al motor con carga, prácticamente sin ningún impacto sobre la velocidad como el que sí se presenta en el arranque suave convencional.

\section{CONCLUSIÓN}

Este artículo ha contribuido y fortalecido el enriquecimiento académico enfocado al control de motores de inducción con rotor jaula de ardilla, presenta el principio de la técnica arranque suave 


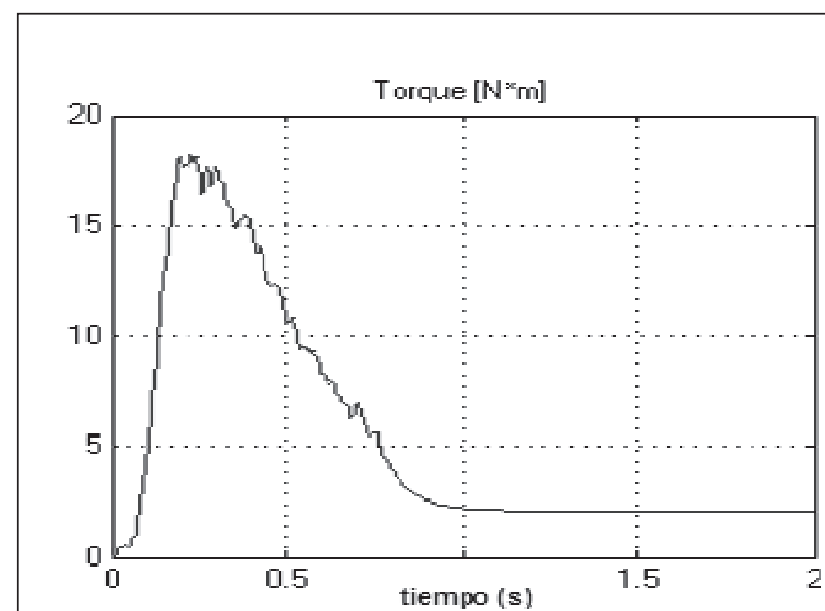

(a)
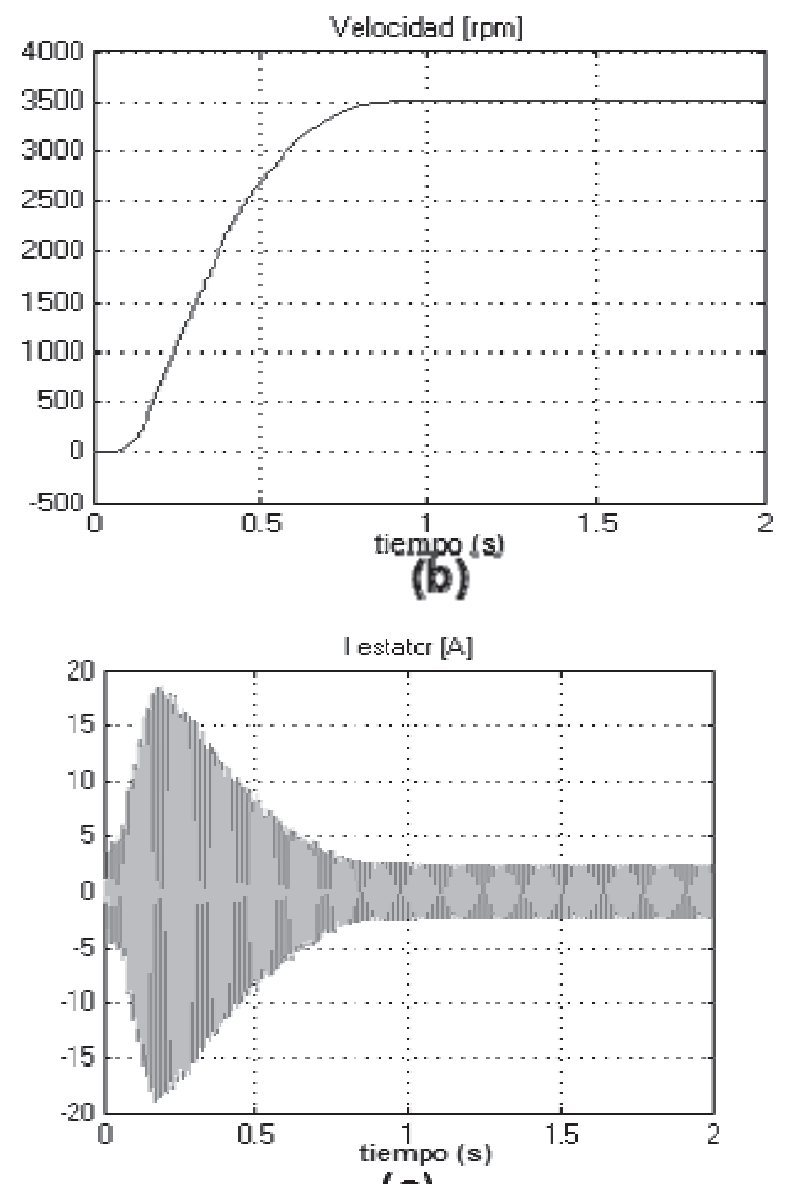

(c)

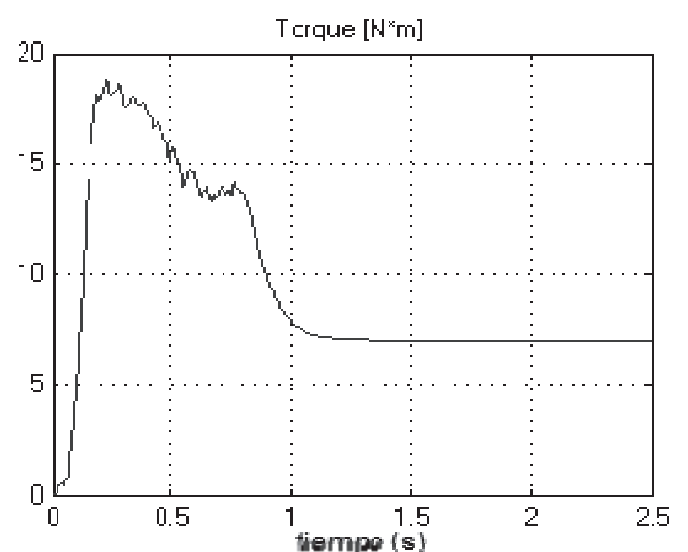

(d)

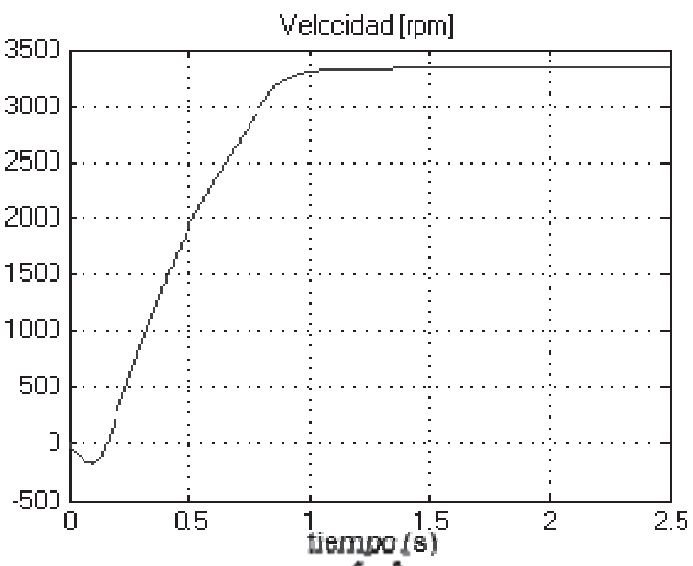

(ब)

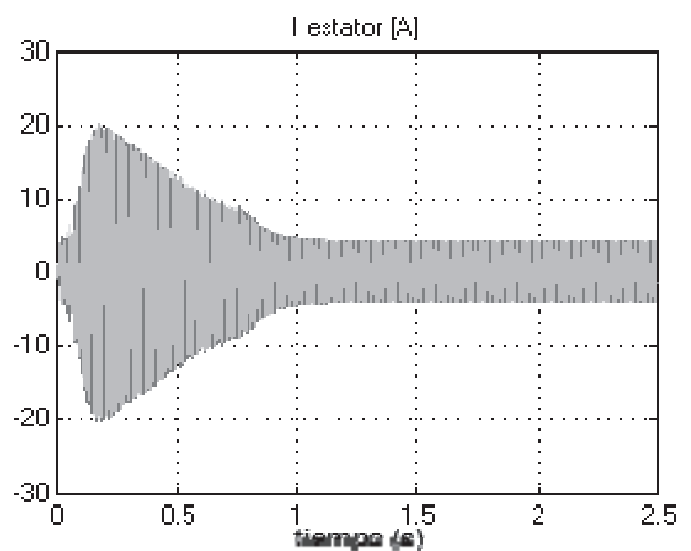

(f)

Fig. 14. Arranque suave difuso en vacío (a) Par, (b) Velocidad, (c) corriente, y con carga 5 N·m. d) Par, (e) Velocidad, (f) corriente. 


\section{con-ciencias}

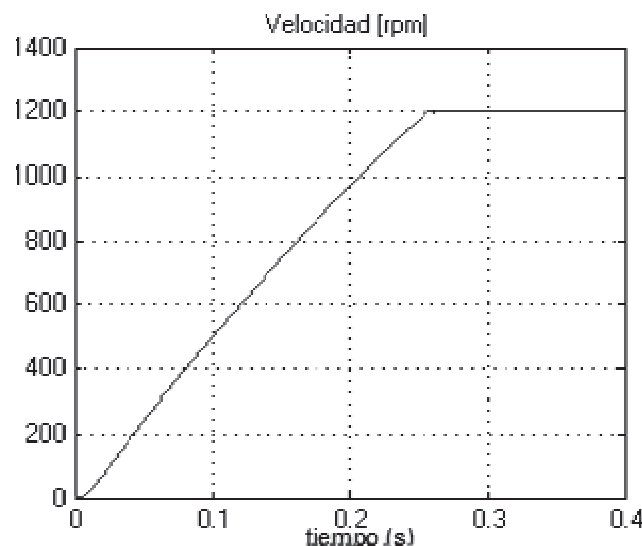

(a)

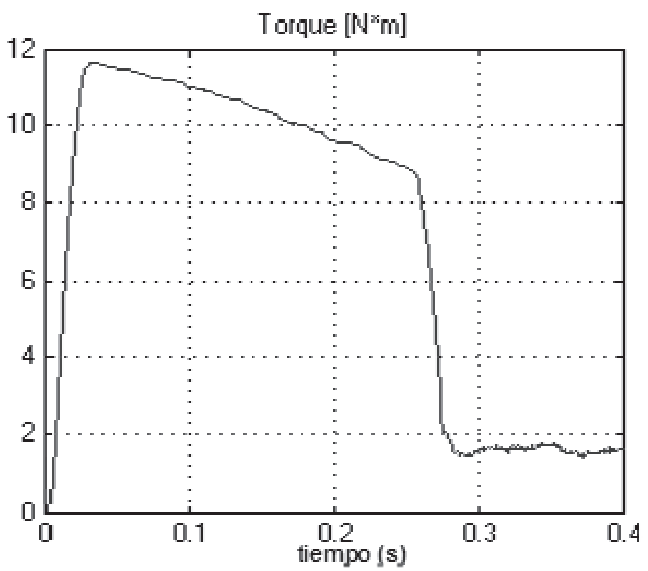

(b)

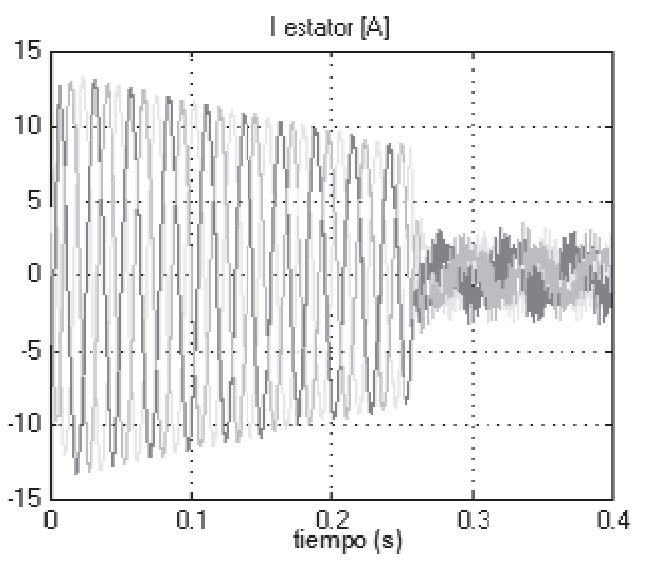

(c)

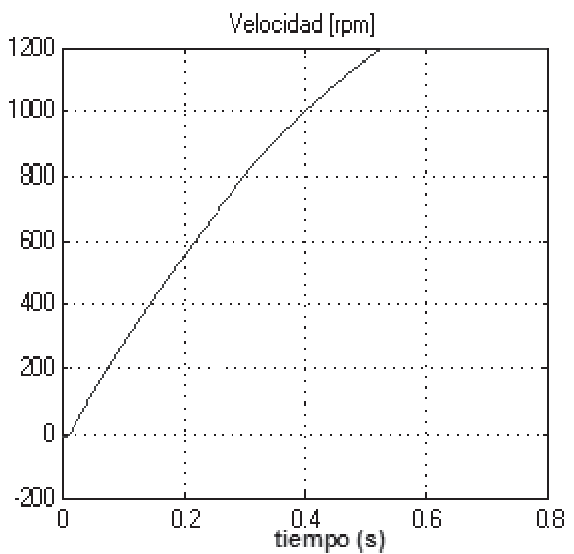

(d)

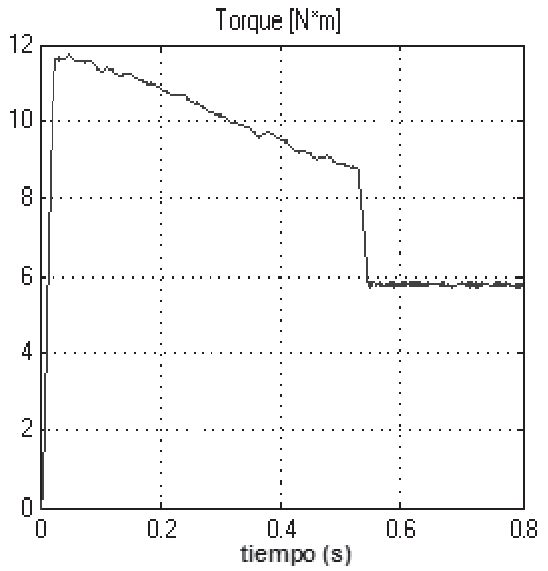

(e)

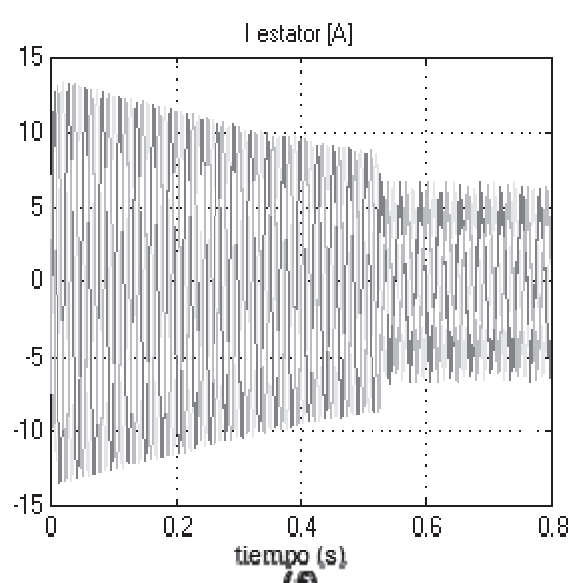

If

Fig. 15. Arranque con el inversor en vacío (a) Velocidad, (b) Par, (c) corriente, y con carga $5 \mathrm{~N} \cdot \mathrm{m}$. d) Velocidad, (e) Par, (f) corriente. 
y control directo de par, incorporando control difuso, implementado mediante dos tipos de arranques electrónicos como son el arranque por tiristores e inversor trifásico.
Los resultados de la simulación muestran un mejoramiento en el desempeño en el periodo de arranque, reduciendo la corriente e incrementando el torque.

\section{REFERENCIAS}

[1] P. Krause. O. Wasynczuk and S. Sudhoff, Analysis of Electric Machinery, McGrawHill, Capítulos 3-4, 1986.

[2] R. McElveen and M. Toney, "Starting high inertia loads," IEEE Transactions Industry Applications, vol. 37, no. 1, pp. 137-144, Jan./Feb. 2001.

[3] S. Sivanandam and S. Sumathi, Introduction to Fuzzy Logic using MatLab, Berlin Heidelberg: Springer-Verlag, 2007.

[4] J. Ramírez, M. Salamanca and O. Higuera, "Control óptimo para el arranque en tiempo mínimo de un motor de inducción”, Tecnura, vol. 13, no. 25, pp. 48-58, Dic. 2009.

[5] L. Ghodhbani, F. Kourda, B. Rebhi and M. Elleuch, "Comparison among electronic start up methods for induction motors," 7th International Multi-Conference on Systems, Signals and Devices. Amman. June. 2010.

[6] L. Rajaji, C. Kumar and M. Vasudevan. "Fuzzy and anfis based soft starter fed in- duction motor drive for high performance applications," ARPN Journal of Engineering and Applied Sciences, vol. 3, no 4. pp. 12-24, Aug. 2008.

[7] Z. Kaiqi, X. Dianguo and W. Yi. "Discrete variable frequency soft starting on DSPbased voltage controller-fed IM drive," Industrial Electronics Society, IECON '03. The 29th Annual Conference of the IEEE, China, Nov. 2003.

[8] P. Vas, Sensorless Vector and Torque Control, United States: Oxford University Press, 1998.

[9] R. Toufouti S. Meziane and H. Benalla, "Direct torque control for induction motor using fuzzy logic," ACSE Journal American Society ok Cicil Engineers, vol. 6, no. 2, pp. 19-26, June. 2006.

[10] M. Baishan, L. Haihua, Z. Jinping. "Study of fuzzy control in direct torque control system," International Conference on Artificial Intelligence and Computational Intelligence, Shanghai. Nov. 2009. 